\title{
Corrigendum to "Hepatic Iron Quantification on 3 Tesla (3 T) Magnetic Resonance (MR): Technical Challenges and Solutions"
}

\author{
Muhammad Anwar $\mathbb{D}^{1},{ }^{1}$ John Wood, ${ }^{2,3}$ Deepa Manwani, ${ }^{4}$ Benjamin Taragin $\mathbb{D},{ }^{1}$ \\ Suzette O. Oyeku, ${ }^{5}$ and Qi Peng ${ }^{1}$ \\ ${ }^{1}$ Department of Pediatric Radiology, Children's Hospital at Montefiore, Bronx, NY, USA \\ ${ }^{2}$ Department of Pediatric Radiology, Children's Hospital Los Angeles, Los Angeles, CA, USA \\ ${ }^{3}$ Division of Pediatric Cardiology, Children's Hospital Los Angeles, Los Angeles, CA, USA \\ ${ }^{4}$ Department of Pediatrics, Division of Pediatric Hematology/Oncology, Children's Hospital at Montefiore, Bronx, NY, USA \\ ${ }^{5}$ Department of Pediatrics, Division of General Pediatrics, Children's Hospital at Montefiore, Bronx, NY, USA
}

Correspondence should be addressed to Muhammad Anwar; masaeed@excite.com

Received 1 March 2018; Accepted 4 March 2018; Published 3 June 2018

Copyright (C) 2018 Muhammad Anwar et al. This is an open access article distributed under the Creative Commons Attribution License, which permits unrestricted use, distribution, and reproduction in any medium, provided the original work is properly cited.

In the article titled "Hepatic Iron Quantification on 3 Tesla (3 T) Magnetic Resonance (MR): Technical Challenges and Solutions" [1], there was an error in equation (2), where the $R_{2}^{*}$ values for $1.5 \mathrm{~T}$ and $3 \mathrm{~T}$ are reversed. The corrected equation is as follows:

$$
R_{2}^{*}\left(1.5 \mathrm{~T}, C_{\mathrm{Fe}}\right)=\frac{\left(R_{2}^{*}\left(3 \mathrm{~T}, C_{\mathrm{Fe}}\right)+11\right)}{2.0} .
$$

\section{References}

[1] M. Anwar, J. Wood, D. Manwani, B. Taragin, S. O. Oyeku, and Q. Peng, "Hepatic iron quantification on 3 tesla (3 T) magnetic resonance (MR): technical challenges and solutions," Radiology Research and Practice, vol. 2013, Article ID 628150, 7 pages, 2013. 


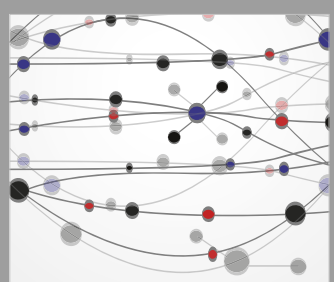

The Scientific World Journal
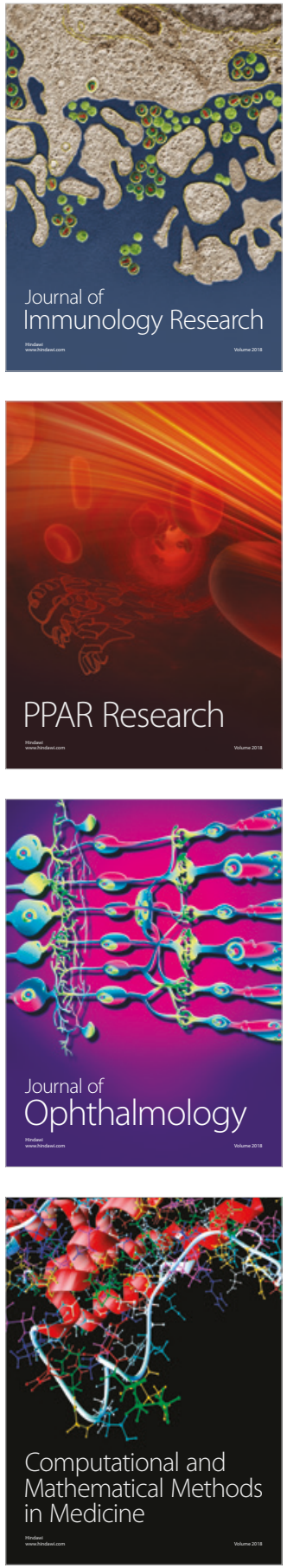

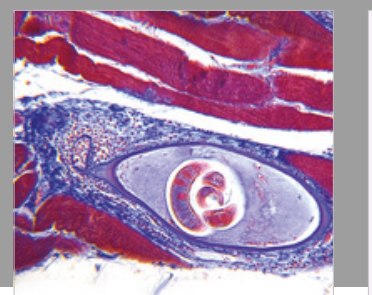

Gastroenterology Research and Practice

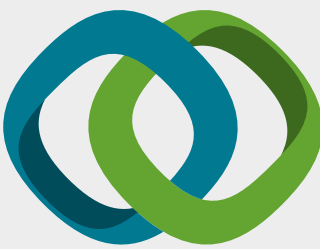

\section{Hindawi}

Submit your manuscripts at

www.hindawi.com
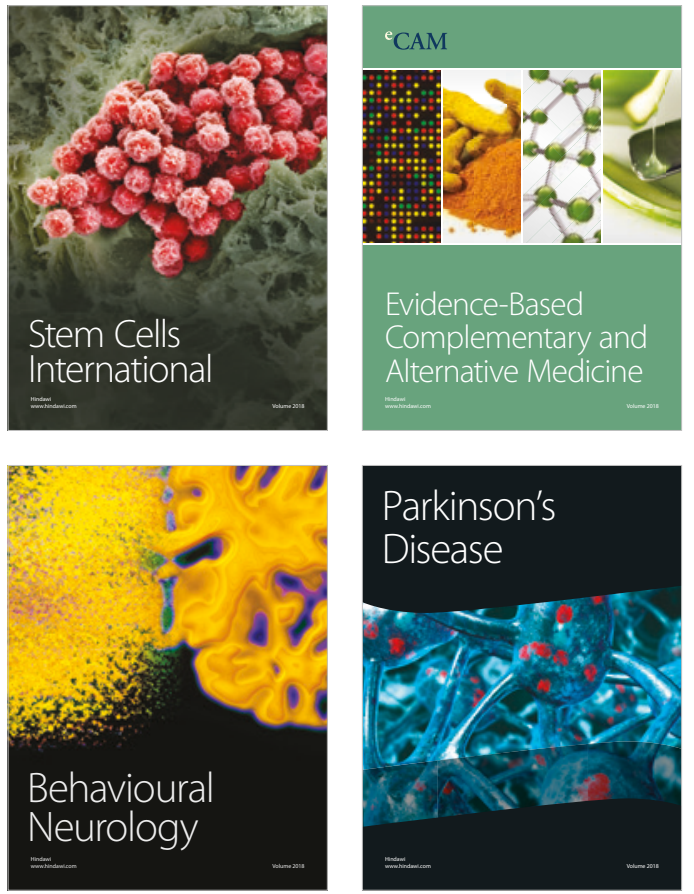

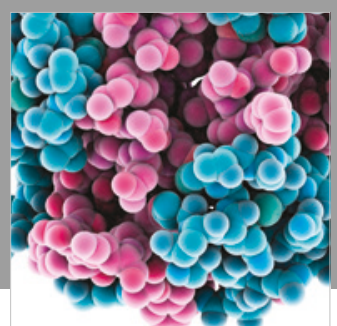

ournal of

Diabetes Research

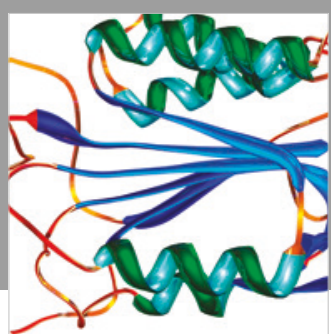

Disease Markers
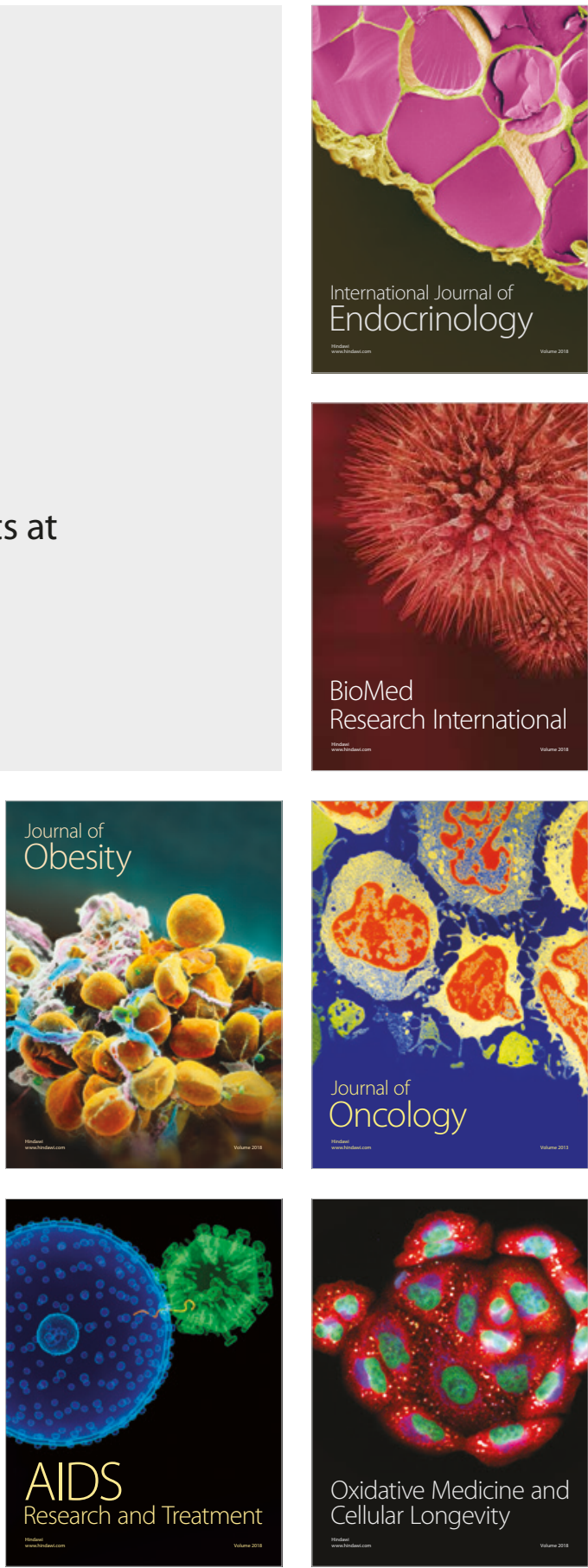